\section{MCMI-III Personality Disorders, Traits, and Profiles in Adult ADHD Outpatients}

Journal of Attention Disorders

2020, Vol. 24(6) 830-839

(C) The Author(s) 2018

Article reuse guidelines:

sagepub.com/journals-permissions DOI: 10.1 I 77//10870547/8780319

journals.sagepub.com/home/jad

(S)AGE

\author{
Francesco Oliva' (D), Chiara Mangiapane', Gabriele Nibbio², \\ Alberto Portigliatti Pomeri', and Giuseppe Maina'
}

\begin{abstract}
Objective: To assess prevalence of personality traits and disorders according to Millon's evolution-based model and to identify the most representative personality profiles among adult ADHD outpatients. Method: Personality traits and disorders were evaluated using the Millon Clinical Multiaxial Inventory-III (MCMI-III) and an exploratory factor analysis (EFA) in a consecutive sample of adult ADHD outpatients $(N=70)$ diagnosed by the Adult ADHD Self-Report Scaleversion I.I (ASRS-vI.I) and the Diagnostic Interview for ADHD in Adults (DIVA 2.0). Results: More than half of our sample (57.1\%) showed at least one personality disorder (PD). The most prevalent PDs were paranoid, schizotypal and negativistic (18.6\% for all three PDs), depressive (I7.I\%), and sadistic (II.4\%). No patient had a borderline PD. The EFA identified three personality profiles ("sadistic-antisocial-negativistic," "masochistic-depressive-dependent-avoidant," and "antihistrionic-schizoid"). Conclusion: High prevalence of PDs among adult ADHD patients was confirmed. The personality profiles seemed to reflect the persistence of ADHD and related childhood comorbidities in adulthood. (J. of Att. Dis. 2020; 24(6) 830-839)
\end{abstract}

\title{
Keywords
}

ADHD, personality disorders, MCMI-III, Millon, personality traits

\section{Introduction}

ADHD is a neurodevelopmental disorder characterized by a persistent pattern of inattention and/or hyperactivity/impulsivity leading to significant functional impairment (American Psychiatric Association [APA], 2013). In addition to the core symptoms of ADHD (i.e., inattention, hyperactivity, and impulsivity), patients also manifest some associated features in their lifetime (APA, 2000, 2013) such as low self-esteem (Cook, Knight, Hume, \& Qureshi, 2014) and emotional dysregulation (Hirsch, Chavanon, Riechmann, \& Christiansen, 2018), which can contribute to significantly affect patients' functioning (Harpin, Mazzone, Raynaud, Kahle, \& Hodgkins, 2016).

The prevalence of ADHD during childhood is estimated to range from $4 \%$ to $8 \%$ with a slight diagnostic prevalence in males (Faraone, Sergeant, Gillberg, \& Biederman, 2003). Although functional impairment recedes in a large number of cases with growth and neural development, this disorder can be considered persistent, as $60 \%$ of patients continue to suffer significant symptoms during adulthood, and some functional impairment persists in $90 \%$ of cases (Biederman, Mick, \& Faraone, 2000). According to a recent international meta-analysis, the cross-national mean prevalence of ADHD in adulthood is $2.8 \%$ (ranging between $0.6 \%$ and $7.3 \%$ depending on country income level), without gender differences (Fayyad et al., 2017).
Of adults with ADHD, 50\% to $75 \%$ are affected by an another psychiatric disorder (Kooij, 2012). One study reported even higher prevalence of comorbidity, reaching 80\% (Klassen, Katzman, \& Chokka, 2010). According to the literature, an adult ADHD patient has an average of three other psychiatric disorders (Fayyad et al., 2017; Kooij et al., 2012).

Personality disorders (PDs) are among the most frequently diagnosed psychiatric disorders in adult patients with ADHD; a recent review reported a prevalence of comorbidity between 10\% and 75\% (Matthies \& Philipsen, 2016).

Personality describes a set of traits that are persistent patterns of perceiving, thinking about, and relating to the surrounding environment and to other people and is expressed in many different contexts, including social and personal. According to the Diagnostic and Statistical Manual of Mental Disorders (5th ed.; DSM-5; APA, 2013), a PD can be defined as an enduring pattern of conduct and inner experience that significantly differs from what is expected in accordance with the individual's culture. A PD

'University of Turin, Italy

${ }^{2}$ University of Brescia, Italy

Corresponding Author:

Francesco Oliva, Department of Clinical and Biological Sciences,

University of Turin, Regione Gonzole 10, 10043 Orbassano, Turin, Italy.

Email: francesco.oliva@unito.it 
is characterized by inflexibility and pervasiveness and arises during adolescence or early adulthood remaining stable over time and causes functional impairment or clinically significant distress (APA, 2013).

Prevalence of PDs varies according to the considered PD; however, cross-sectional epidemiological surveys reported a point prevalence of PDs ranging from $4 \%$ to $15 \%$ (Tyrer, Reed, \& Crawford, 2015), depending on the country where the study was conducted (i.e., $13.4 \%$ in Norway, Torgersen, Kringlen, \& Cramer, 2001; $15 \%$ in the United States, Grant et al., 2004; 4.4\% in Great Britain, Coid, Yang, Tyrer, Roberts, \& Ullrich, 2006).

Data regarding the prevalence of comorbidity between ADHD and PDs are obtained mostly from evaluations based on categorical models, such as that described in the DSM (Anckarsäter et al., 2006; Bernardi et al., 2012; Edvinsson, Lindström, Bingefors, Lewander, \& Ekselius, 2013). In particular, a strong association between adult ADHD and dramatic, overly emotional, erratic PDs included in the cluster B of DSM (i.e., antisocial, borderline, histrionic and narcissistic PDs; APA, 2000, 2013) emerged in several studies (Fossati, Novella, Donati, Donini, \& Maffei, 2002; Kooij, 2006; Philipsen et al., 2008; van Dijk, Lappenschaar, Kan, Verkes, \& Buitelaar, 2011). High chances of developing a cluster B PD were also been shown by adolescents with ADHD: Borderline (odds ratio $[\mathrm{OR}]=13.16$ ), antisocial $(\mathrm{OR}=3.03)$, and narcissistic $(\mathrm{OR}=8.69)$ PDs (Miller et al., 2008). Therefore, according to the literature, borderline PD may be the most frequent PD in adult ADHD patients.

Although some authors have already investigated the prevalence of personality traits and disorders among adult ADHD patients using a dimensional approach, mostly Cloninger's model (Faraone, Kunwar, Adamson, \& Biederman, 2009; Salgado et al., 2009), only a few have used the Millon's evolution-based model to assess personality in ADHD patients (Gudjonsson, Wells, \& Young, 2012; Hamzeloo, Mashhadi, \& Salehi Fadardi, 2016; May \& Bos, 2000; McKinney, Canu, \& Schneider, 2013; Salavera et al., 2014).

According to Millon's theory, personality can be characterized using three polarities (pleasure-pain, active-passive, and self-other) derived from four ecological principles (aims of existence, modes of adaptation, strategies of replication, and processes of abstraction). Therefore, each of the 14-personality prototypes identified by Millon can be strongly, weakly, or neutrally placed in any of these three polarities. Starting from his dimensional approach to psychopathology, Millon developed the Millon Clinical Multiaxial Inventory (MCMI), an assessment tool that allows a comprehensive evaluation of both personality and clinical syndromes. One of the strengths of the MCMI is that it does not only identify PDs, as categorical diagnostic entities (mostly in accordance to the DSM-IV classification), but it also provide a dimensional estimation of personality traits and PD severity (Choca, 2004; Choca \& Grossman, 2015).
In literature, the few studies using MCMI to assess personality in patients affected by adult ADHD are characterized by setting and method heterogeneity, and they also show some limitations. Four studies (Gudjonsson et al., 2012; Hamzeloo et al., 2016; McKinney et al., 2013; Salavera et al., 2014) are conducted in specific subpopulations; thus, they cannot be considered representative of the general population. Really high prevalence of antisocial $(100 \%)$, borderline $(100 \%)$, avoidant $(59.1 \%)$, dependent (59.1\%), schizotypal (59.1\%), and negativistic (45.5\%) PDs were found using the MCMI-II (i.e., the second edition of MCMI) among 22 Spanish homeless people who positively screened for adult ADHD (Salavera et al., 2014). Two studies were conducted on prisoners affected by adult ADHD using the MCMI-III (i.e., the third edition of MCMI), reporting a high prevalence of antisocial (44.2\%), borderline (21\%), depressive (20.4\%), negativistic (10.2\%), and dependent $(9.5 \%)$ PDs in an Iranian sample $(N=147$; Hamzeloo et al., 2016) and a negative correlation between compulsive scale elevation and ADHD symptoms in a Scottish sample $(N=26$; Gudjonsson et al., 2012). Eventually, a study using only three scales of the MCMI-III (i.e., antisocial, compulsive, masochistic) was conducted on university students without ADHD diagnoses $(N=170)$ and did not report any prevalence of PDs, but revealed a strong association between ADHD symptoms and elevations on MCMI-III scales (McKinney et al., 2013).

To the best of our knowledge, there is only one study assessing personality in a clinical sample of adults affected by ADHD $(N=104)$ using MCMI-II, in which prevalence of prominent personality characteristics was stratified, accounting for comorbidities (May \& Bos, 2000). This study revealed important variation in prevalence of personality prototypes in patients having a comorbid oppositional defiant disorder and even more so when they also presented other psychiatric comorbidities. However, the authors, despite the MCMI-II manual indications, made no distinction between personality traits and disorders, and thus the actual prevalence of PDs in adult ADHD patients could not be determined.

Furthermore, there were no studies applying a factorial analysis model to the MCMI dimensional assessment in a sample of ADHD patients, although findings about application of both exploratory factor analysis (EFA) and confirmatory factor analyses (CFAs) on MCMI have been already reported (Cuevas, García, Aluja, \& García, 2008) and this particular type of statistical technique is expressively designed to inform about the underlying structure of specific phenomena (Wright, 2017).

The aim of the present study was to assess prevalence of personality traits and disorders according to Millon's evolution-based model and to identify the most representative personality profiles, as determined by EFA, in a clinical sample of outpatients affected by adult ADHD. 


\section{Method}

\section{Sample and Enrollment}

Patients were recruited as a consecutive sample at the adult ADHD outpatient center of the AOU San Luigi Gonzaga (Orbassano, Turin, Italy), from January 2015 to May 2017. All adult patients accessing the adult ADHD center were screened using the Adult ADHD Self-Report Scale-version 1.1 (ASRS-v1.1) on the first psychiatric examination, and their sociodemographic and clinical features were collected through an ad hoc clinical record (i.e., age, gender, education, employment, family history of psychiatric disorders, lifetime medical and psychiatric comorbidity, type of psychiatric comorbidity). During the psychiatric examination, a psychiatrist performed a comprehensive evaluation of the patients' overall psychopathological condition, even assessing psychiatric comorbidity. A trained psychiatrist then tested patients with positive ASRS v-1.1 using the Diagnostic Interview for ADHD in Adults (DIVA 2.0) to confirm the diagnosis according to $D S M-I V$ criteria.

The study inclusion criteria required participants to (a) be $\geq 18$ years and (b) have a diagnosis of ADHD according to $D S M-I V$ criteria. Patients with less than 8 years of education were excluded because they could not meaningfully undertake the MCMI-III, according to the Interpretive Guide to the Millon Clinical Multiaxial Inventory (Choca, 2004). All patients meeting eligibility criteria were asked to participate in the study and sign a written informed consent. Finally, all enrolled patients were assessed using the MCMI-III by either a trained psychologist or psychiatrist. The interpretation of each MCMI-III profile has been performed according to the procedures described in the manual and its Italian validation (Zennaro, Ferracuti, Lang, \& Sanavio, 2008).

\section{Assessment Tools}

The ASRS-v1.1 is a self-administered, internationally validated (Kessler et al., 2005) screening tool for adult ADHD developed by World Health Organization (WHO). It consists of a six-item checklist concerning adult ADHD symptoms. Patients indicate the frequency of occurrence for each symptom, and the screening can be considered positive when at least four answers are above the significance cutoff value.

The DIVA 2.0 is a validated (Ramos-Quiroga et al., 2019) structured interview for the assessment of adult ADHD according to DSM-IV diagnostic criteria. It consists of 18 questions (nine concerning inattentive symptoms and nine about hyperactivity/impulsivity) investigating DSM-IV symptoms (criterion A) by concrete examples, for both childhood and adulthood, to help the patient recognize symptoms. $D S M-I V$ criterion B (i.e., presence of some symptoms before 7 th year of age) and criterion $\mathrm{E}$ (i.e., the symptoms cannot be better explained by the presence of another psychiatric disorder) were both evaluated by explicit questions. The DIVA also provides a section concerning the impact of ADHD symptoms on different areas of the patient's functioning (criterion $\mathrm{C}$ and criterion D). According to DSM- $I V$ and to DIVA criteria, to formulate an adult ADHD diagnosis, the patient must present at least six out of the nine listed symptoms in either of the two clusters or in both (inattention and/or hyperactivity/impulsivity). This must be associated with functioning impairment in at least two areas (work/education; relationship and/or family; social contacts; free time/hobby; self-confidence/self-image). Moreover, the clinician should specify the type of collateral information acquired and the level of its support. All DIVA interviews of the present study were administered with both parental and school reports support to guarantee the highest level of accuracy.

The MCMI-III is a self-report personality inventory developed on Millon's evolution-based personality theory: Citing basic laws of evolution, Theodore Millon described the core motivating aims belonging to evolutionary biology and their connection to personality, which are arranged along three polarities: Existence (pleasure-pain), adaptation (active-passive), and replication (self-other). Different combinations of these motivating aims, then, give rise to a unique personality strategy (Zennaro et al., 2008). The MCMI-III has been also validated in its Italian version (Zennaro et al., 2008). This assessment tool consists of 175 dichotomous (true or false) items for a total amount of 28 scales, including 14 PDs and 10 clinical syndrome scales. The MCMI-III also provides one validity scale (V) and three modifying indices, $\mathrm{X}$-disclosure, $\mathrm{Y}$ - desirability, and $\mathrm{Z}$-debasement index, that assess the response sets of each patient and identify those who are unwilling or unable to read, understand, and complete the inventory appropriately. Millon distinguishes 11 moderate PD scales (i.e., schizoid, avoidant, depressive, dependent, histrionic, narcissistic, antisocial-sadistic compulsive, negativistic, masochistic) and three severe personality pathology scales (i.e., schizotypal, borderline, paranoid) that represent intrinsically more pathological personality prototypes. Clinical syndrome scales (i.e., anxiety, somatoform, bipolar: manic, dysthymia, alcohol dependence, drug dependence, posttraumatic stress disorder, thought disorder, major depression, delusional disorder) assess the clinical symptoms, and they can be used to identify the likely presence of an Axis I disorder according to $D S M-I V$. A useful feature of MCMI-III scales is that they can be interpreted both categorically and dimensionally. After computing the raw scores, they must be converted into standardized scores. The MCMIIII uses the base rate (BR) score to indicate the probability that a specific individual is similar to the group of psychiatric patients with a diagnosis of a particular trait or disorder. According to the MCMI-III manual and therefore to the prevalence of each PDs in the normative sample (Strack \& Millon, 2007; Zennaro et al., 2008), a BR score of a moderate PD scale ranging between 75 and 84 defines the presence of a personality trait, whereas a score $\geq 85$ suggests that the patient 
is affected by a PD (i.e., a $D S M-I V$ PD when the PD scale considered corresponds to a $D S M-I V$ PD). Conversely, when accounting for severe personality pathology scales, it is possible to diagnose a PD with a score above 75 , as they represent intrinsically pathological personality prototypes. Psychometric properties have already been confirmed and in particular the evaluation of MCMI-III reliability revealed that test-retest coefficient of almost all personality scales is above 0.8 (Wise, Streiner, \& Walfish, 2010). All BR scores were adjusted for modifying indices according to the Italian manual and validation (Zennaro et al., 2008).

\section{Statistical Analysis}

All computations were performed using the IBM SPSS Statistics for MACOS package (version 22.0, IBM Corporation, Armonk, NY, USA). The descriptive analysis of the sample included the frequencies of categorical variables and the calculation of means and standard deviations for normally distributed continuous variables and medians and interquartile ranges for nonnormally distributed variables. Normal distribution was tested using a Shapiro-Wilk test.

Personality traits and disorders of each participant were evaluated using BR thresholds according to MCMI-III manual and its Italian validation, and then, we estimated the prevalence of each trait and disorder in the sample. We also calculated the number of disorders per patient.

To identify some personality profiles (i.e., correlation between different personality scale scores) among our sample of adult ADHD outpatients, we conducted an EFA on MCMI-III Axis II scales using BR scores as continuous variables and the principal axis factor as methods of extraction. Taking into account the relative small sample to variable ratio $(N: p$ ratio $=5: 1)$, the suitability of the respondent data returned by the EFA model was assessed by both Kaiser-Meyer-Olkin (KMO) Measure of Sampling Adequacy and Bartlett's test of sphericity.

The number of factors retained was determined by both Kaiser's criterion (eigenvalue $>1$ ) and a scree plot test (factors retained above the break point from a straight line drawn through the smaller eigenvalues on a scree plot, not including the break point itself). Data interpretation was formulated using a varimax orthogonal rotation with Kaiser's normalization and considering only personality traits with a coefficient above 0.6. Each factor was labeled using the name of the involved traits and placing "anti" before the name when the coefficient was negative.

\section{Results}

\section{Sample Characteristics}

Out of 107 evaluated patients, 70 were diagnosed with adult ADHD according to the $D S M-I V$ criteria. As no patient met
Table I. Sociodemographic and Clinical Features of the Study Sample $(N=70)$.

\begin{tabular}{lc}
\hline & $n(\%$ of sample) \\
& or $M( \pm S D)$ \\
\hline Age, years & $30.8( \pm 11.53)$ \\
Gender, male & $49(70 \%)$ \\
Education, years & $13.3( \pm 3.39)$ \\
Employment, yes & $33(47.1 \%)$ \\
Family history of & \\
ADHD & $17(24.3 \%)$ \\
Other psychiatric disorders & $28(40 \%)$ \\
Lifetime medical comorbidity, yes & $24(34.3 \%)$ \\
Lifetime psychiatric comorbidity, yes & $50(71.4 \%)$ \\
Psychiatric comorbidity & \\
Any depressive disorder & $16(22.8 \%)$ \\
Any anxiety disorder & $8(11.4 \%)$ \\
Any bipolar disorder & $17(24.3 \%)$ \\
Obsessive-compulsive disorder & $1(1.4 \%)$ \\
Alcohol use disorder & $13(18.6 \%)$ \\
Substance use disorder & $34(48.5 \%)$ \\
Personality disorders & $5(7.1 \%)$ \\
Oppositional defiant disorder & $25(35.7 \%)$ \\
ASRS-vI.I & $4.7( \pm 0.94)$ \\
DIVA 2.0 & \\
Attention deficit during childhood & $7.1( \pm 1.78)$ \\
Attention deficit during adulthood & $7.6( \pm 0.93)$ \\
Hyperactivity/impulsivity during childhood & $4.5( \pm 3.28)$ \\
Hyperactivity/impulsivity during adulthood & $4.5( \pm 2.58)$ \\
ADHD type during childhood & $34(48.6 \%)$ \\
Predominantly inattentive & $36(51.4 \%)$ \\
Predominantly hyperactive/impulsive & $3(4.3 \%)$ \\
Combined & $31(44.3 \%)$ \\
ADHD type during adulthood & $33(47.1 \%)$ \\
Predominantly inattentive & $3(4.3 \%)$ \\
Predominantly hyperactive/impulsive & \\
Combined & \\
\hline
\end{tabular}

Note. ASRS $=$ ADHD Self-Report Scale; DIVA = Diagnostic Interview for ADHD in Adults.

the exclusion criterion, they were all included in the study. Sociodemographic and clinical features of the sample are presented in Table 1.

\section{Personality Traits and Disorders}

According to the validity scale scores, all MCMI-III tests were deemed valid $(100 \% ; N=70$ with V scale $=0)$.

Among the 70 recruited patients, $42.8 \%(n=30)$ did not show any PD, $18.6 \%(n=13)$ had one PD, $18.6 \%(n=13)$ had two PDs, and $20.0 \%(n=14)$ had three or more PDs.

Figure 1 shows the prevalence of each type of personality trait and disorder within our sample.

The most represented personality trait was negativistic $(45.7 \% ; n=32)$, followed by depressive $(30 \% ; n=21)$; 


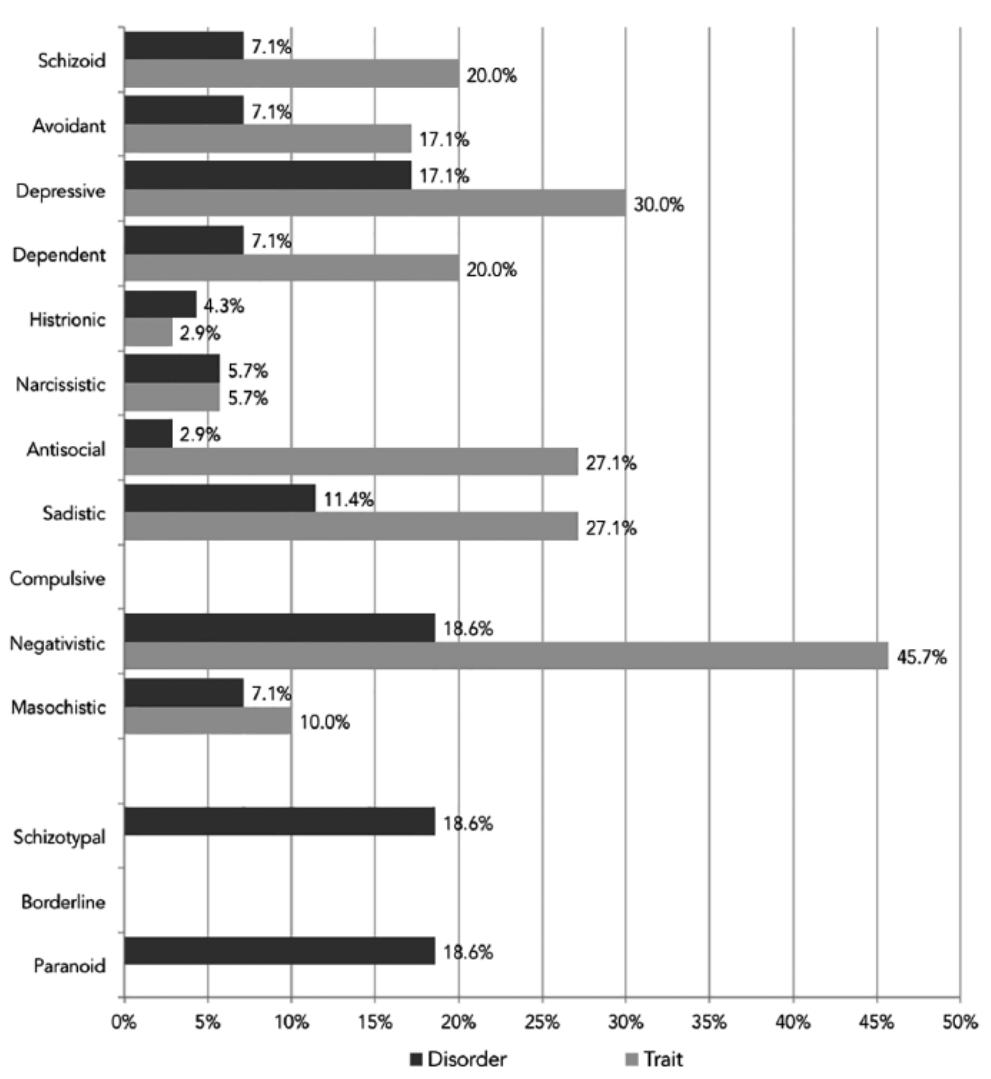

Figure I. Prevalence of MCMI-III personality traits and disorders $(N=70)$. Note. MCMI-III = Millon Clinical Multiaxial Inventory-III.

antisocial and sadistic traits had the same prevalence of $27.1 \%$ ( $n=19$ for each).

With regard to PDs, among the so-called severe personality pathologies, paranoid and schizotypal PDs shared the same prevalence $(18.6 \% ; n=13)$, whereas no patient was affected by borderline PD. Among the moderate PDs, the most frequent was negativistic $(18.6 \% ; n=13)$, followed by depressive $(17.1 \% ; n=12)$ and sadistic $(11.4 \% ; n=8)$. No compulsive PDs were found (Figure 1).

\section{$E F A$}

Assessment of sampling adequacy showed that data were suitable for factor analysis, $\mathrm{KMO}=0.815$; Bartlett's test, $\chi^{2}(d F)=541.2(91), p<.001$. Three factors met Kaiser's criterion showing an eigenvalue $>1$ and cumulatively explained $67.7 \%$ of the total variance.

Similarly, looking at the scree plot, the break point from the line passing through smaller eigenvalues was the Factor 4 (Figure 2). Therefore, the first three factors were retained in factor analysis. The factor matrix resulting from the varimax rotation is presented in Table 2. The adoption of a

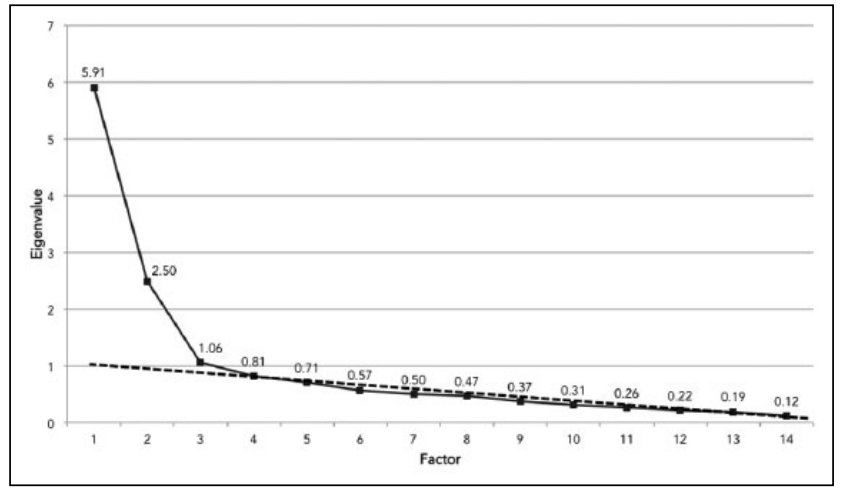

Figure 2. Scree plot of the exploratory factor analysis.

threshold of 0.6 reduced as much as possible the presence of the same trait in different factors.

The first factor explained $42.2 \%$ of variance and presented a high positive correlation between BR scores on sadistic, antisocial, and negativistic scales, so it has been labeled as "sadistic-antisocial-negativistic" factor. The second factor explained $17.8 \%$ of the variance and was characterized by a 
Table 2. Rotated Factor Matrix of the Exploratory Factor Analysis $(N=70)$.

\begin{tabular}{lccc}
\hline & \multicolumn{3}{c}{ Factor } \\
\cline { 2 - 4 } MCMI-III scale & \multicolumn{1}{c}{2} & 2 & \multicolumn{1}{c}{3} \\
\hline Schizoid & 0.027 & -0.062 & $\mathbf{0 . 7 1 6}$ \\
Avoidant & 0.023 & $\mathbf{0 . 6 0 6}$ & 0.591 \\
Depressive & 0.332 & $\mathbf{0 . 6 3 1}$ & 0.186 \\
Dependent & 0.257 & $\mathbf{0 . 6 4 2}$ & 0.257 \\
Histrionic & -0.154 & -0.272 & $-\mathbf{0 . 8 5 3}$ \\
Narcissistic & 0.342 & -0.311 & -0.540 \\
Antisocial & $\mathbf{0 . 7 4 3}$ & 0.098 & -0.046 \\
Sadistic & $\mathbf{0 . 8 6 3}$ & 0.273 & 0.034 \\
Compulsive & -0.540 & -0.238 & -0.097 \\
Negativistic & $\mathbf{0 . 7 0 2}$ & 0.480 & 0.010 \\
Masochistic & 0.305 & $\mathbf{0 . 7 0 0}$ & 0.068 \\
Schizotypal & 0.321 & 0.429 & 0.454 \\
Borderline & 0.493 & 0.488 & 0.039 \\
Paranoid & 0.545 & 0.533 & 0.188 \\
\hline
\end{tabular}

Note. Extraction method: principal axis factoring. Rotation method: varimax with Kaiser normalization. Rotation converged in five iterations. Coefficients above 0.6 are indicated in bold. MCMI-III = Millon Clinical Multiaxial Inventory-III.

positive correlation between masochistic, depressive, dependent, and avoidant BR scores, thus it has been labeled as "masochistic-depressive-dependent-avoidant" factor. Finally, the third factor explained $7.6 \%$ of the variance and was positively correlated with histrionic and negatively correlated with schizoid BR scores (Table 2). This last factor has been labeled "antihistrionic-schizoid."

\section{Discussion}

The prevalence of PDs among our sample of adult ADHD outpatients was consistent with those reported by previous epidemiological and clinical studies, as more than half of the sample presented at least one PD. Although an in-depth comparison with previous studies is difficult due to differences in setting (i.e., in- vs. out-patients), enrollment (e.g., recruitment by general population vs. clinical sample with other psychiatric disorders), and personality assessment tools (i.e., categorical vs. dimensional), it is possible to conclude that the prevalence of PDs in ADHD adults is consistently higher (10\%-75\%, Matthies \& Philipsen, 2016) than that reported in the general population (4\%-15\%, Tyrer et al., 2015).

Thanks to the intrinsic characteristics of the MCMI-III and careful observation of the indications given by its manual, we evaluated the prevalence of each type of PD. The three severe PDs (i.e., paranoid, borderline, schizotypal) were distinguished by Millon because they are structurally defective PDs considered as more severe compared with the other personality prototypes (Choca, 2004; Millon \& Grossman, 2007a).
One of the main unexpected findings of the present study is that no patient had a borderline PD, whereas both paranoid and schizotypal PDs reached a high prevalence in our sample. Previous studies using MCMI conducted on either prison inmates (Hamzeloo et al., 2016) or homeless people (Salavera et al., 2014) reported a considerably higher prevalence of borderline PD compared with paranoid and schizotypal PDs. This discrepancy could be explained by recruitment differences in setting (outpatients vs. prisoners or homeless people) and gender (both genders vs. male only). Moreover, the diagnosis of $D S M-I V$ ADHD seems to be more accurate in the present study as it was based on a systematic assessment including ASRS as screener and DIVA, supported by collateral retrospective information, as diagnostic interview than in previous ones, which used only screening tools (Gudjonsson et al., 2012; Hamzeloo et al., 2016) or symptoms scales (McKinney et al., 2013), or a combination of both (Salavera et al., 2014). According to the latest developments in the Millon's theory (Choca \& Grossman, 2015; Millon \& Grossman, 2007a), paranoid PD is frequently encountered together with other moderate PDs (more frequently antisocial-sadistic and narcissistic PDs). This could be interpreted as sign of severe constriction and inflexibility of the overall personality profile, whereas schizotypal PD should be seen as a more serious type of schizoid and avoidant PDs, conferring greater detachment to the already impoverished social life.

Severe PDs therefore must be considered together with the moderate ones to be fully understood (Millon \& Grossman, 2007a, 2007b). The present study found a high rate of several moderate traits and PDs, and among these, the prototypes not listed in the DSM reached the higher prevalence. In particular, almost half of the sample showed negativistic traits, while a third presented depressive traits. These traits were so severe that they were PDs in more than one sixth of the sample. According to Millon's theory, the negativistic prototype presents several features that resemble not only ADHD symptoms but also the frequently comorbid oppositional defiant disorder ( $8.3 \%$ in adult ADHD, Fayyad et al., 2017; 43\% of adult ADHD have a history of oppositional defiant disorder, Harpold et al., 2007). Patients with negativistic personality are typically expressively resentful (e.g., resist fulfilling expectations of others, frequently exhibiting procrastination), interpersonally contrary (e.g., assume conflicting and changing roles in social relationships, particularly dependent and contrite acquiescence, and assertive and hostile independence), and cognitively skeptical (e.g., cynical, doubtful, and untrusting). They also have an irritable mood and frequently show impulsive-like behavior, discharging anger and other troublesome emotions precipitously (Choca, 2004; Millon \& Grossman, 2007b).

Conversely, the depressive personality is fundamentally characterized by self-criticism and extremely low selfesteem, which portrays a sense of permanent hopelessness 
and pessimism (Millon \& Grossman, 2007a). Low selfesteem is commonly observed in adult patients with ADHD (Cook et al., 2014; Newark, Elsässer, \& Stieglitz, 2016) to such extent that it was considered one of the accessory symptoms of adult ADHD (APA, 2000).

Another important dimension was outlined by high prevalence of antisocial and sadistic traits and disorders. According to the MCMI-III and its following interpretative manuals (Choca, 2004; Craig, 2005), all Axis-II B scales have been introduced as more severe versions of A scales. In particular, the sadistic personality is a more pathological variant of the antisocial $\mathrm{PD}$, which, in turn, has been developed to meet $D S M-I V$ criteria for a disorder. The sadistic personality trait describes the subject as assertively competitive, whereas in the disorder, the subject is remarkably detached from an awareness of the impact of their own destructive acts to such an extent that it can be described as callous or cold-blooded (Millon \& Grossman, 2007b). The antisocial personality is instead characterized by a high level of nonconformity and impulsiveness (Millon \& Grossman, 2007b). The antisocialsadistic dimension could be really similar to and maybe derived from conduct disorder in childhood, as well recognized by the DSM classification (APA, 2000, 2013). Moreover, some previous papers have already supported the strong relationship between childhood conduct disorder and antisocial PD in adulthood in patients with ADHD (Biederman, Petty, Dolan, et al., 2008; Biederman, Petty, Monuteaux, et al., 2008; Dowson, 2008).

Considering the high level of PD comorbidity, the attempt to describe some personality profiles of adult ADHD using EFA has proven to be very enlightening, to such an extent that it can be considered the greatest strength of the present study. Three profiles matching the same number of factors have been identified. They seem to depict the three most common ways to adjust to ADHD as a neurodevelopmental disorder, reflecting the persistence of core and accessories symptoms of ADHD and of ingrained common childhood behavioral comorbidities in adulthood. The "sadistic-antisocial-negativistic" profile explained the greatest amount of variance. As mentioned above, elevations on sadistic, antisocial, and negativistic scales were associated with an increase in impulsivity and other typical behaviors of both conduct and oppositional defiant disorders. Each scale of the "masochistic-depressive-dependentavoidant" profile is tied to a different way to deal with lack of self-esteem. Applied to the clinical description of ADHD, this profile would seem to convey all manifestations of typical low self-esteem experienced by adult ADHD patients, from self-injury and self-defeating behavior to living in uncertainty and mistrust in their own abilities. Finally, a minor contribution is due to the "antihistrionic-schizoid" profile, which includes detachment and withdrawal from interpersonal relationships and reality. This profile seems to better fit with core features of prevalent inattentive type of ADHD, in which predominance of default mode network activity, in absence of hyperactivity, could lead the patient to spend more time in spontaneous mind-wandering (Kajimura, Kochiyama, Nakai, Abe, \& Nomura, 2016; Kane et al., 2016; Seli, Smallwood, Cheyne, \& Smilek, 2015). These findings could have a direct repercussion on the clinical approach to adult ADHD patients. As these three distinct personality profiles can be routinely identified with the use of the MCMI-III, clinicians could utilize this assessment tool to develop a personalized psychotherapy approach for each patient.

As we aimed to provide an in-depth personality description of a representative sample of adult ADHD outpatients, the study design did not exclude comorbid psychiatric disorders. This is the main limitation of the present study, because previous literature concerning personality among bipolar and major depressive disorders reported different elevation of MCMI-III scales related to the type of mood episode (Choca, 2004). Furthermore, the present study aimed to provide only a preliminary EFA of personality profiles among adult ADHD patients, without any further subanalysis. One limitation that can be underlined is that the sample size was not large enough to perform any ancillary analysis (e.g., comparison between ADHD subtypes). Moreover, another limitation is that our findings may be generalized to adult ADHD outpatients only.

Future studies may have to discern between ADHD subtypes and collect early comorbidity data in a greater sample to investigate possible relationships between personality traits in adulthood and childhood comorbidity and ADHD subtypes by stratified subanalyses. Furthermore, future research could evaluate whether an early treatment of ADHD in childhood may modify and improve the development of personality, and whether a personalized personality-oriented psychotherapy approach, as suggested by Millon and Grossman (Millon \& Grossman, 2007a, 2007b), can be useful in adult ADHD patients.

\section{Conclusion}

The main aim of the present study was to use Millon's evolution-based model to describe the personality of adult ADHD outpatients. Use of the MCMI-III in conformity with its manual indication together with careful interpretation backed by the latest development of Theodore Millon's theory gave consistent findings about the high prevalence of PDs among adult ADHD patients. Moreover, a preliminary explorative factor analysis identified three personality profiles; each seems to reflect the persistence of core and accessories symptoms of ADHD and of ingrained common childhood behavioral comorbidities (i.e., oppositional defiant and conduct disorders) in adulthood. A personalized personality-oriented psychotherapy approach may be useful 
to improve adjustment to ADHD as a neurodevelopmental disorder and, thus, to ameliorate the overall clinical condition of adult ADHD patients.

\section{Acknowledgments}

Researchers would like to thank all patients involved in the study for their valuable contribution.

\section{Author Contributions}

F.O. and C.M. conceived the study and drafted the manuscript. A.P.P. and C.M. collected the data. F.O. and G.N. performed the statistical analysis. G.M. participated in the design and coordination of the study. All authors read and approved the final manuscript.

\section{Declaration of Conflicting Interests}

The author(s) declared no potential conflicts of interest with respect to the research, authorship, and/or publication of this article.

\section{Funding}

The author(s) received no financial support for the research, authorship, and/or publication of this article.

\section{ORCID iD}

Francesco Oliva iD https://orcid.org/0000-0002-1939-552X

\section{References}

American Psychiatric Association. (2000). Diagnostic and statistical manual of mental disorders (4th ed., text rev.). Washington, DC: Author.

American Psychiatric Association. (2013). Diagnostic and statistical manual of mental disorders (5th ed.). Arlington, VA: American Psychiatric Publishing.

Anckarsäter, H., Stahlberg, O., Larson, T., Hakansson, C., Jutblad, S. B., Niklasson, L., . . Rastam, M. (2006). The impact of ADHD and autism spectrum disorders on temperament, character, and personality development. The American Journal of Psychiatry, 163, 1239-1244.

Bernardi, S., Faraone, S. V., Cortese, S., Kerridge, B. T., Pallanti, S., Wang, S., \& Blanco, C. (2012). The lifetime impact of attention deficit hyperactivity disorder: Results from the National Epidemiologic Survey on Alcohol and Related Conditions (NESARC). Psychological Medicine, 42, 875-887.

Biederman, J., Mick, E., \& Faraone, S. V. (2000). Age-dependent decline of symptoms of attention deficit hyperactivity disorder: Impact of remission definition and symptom type. The American Journal of Psychiatry, 157, 816-818.

Biederman, J., Petty, C. R., Dolan, C., Hughes, S., Mick, E., Monuteaux, M. C., \& Faraone, S. V. (2008). The longterm longitudinal course of oppositional defiant disorder and conduct disorder in ADHD boys: Findings from a controlled 10-year prospective longitudinal follow-up study. Psychological Medicine, 38, 1027-1036.
Biederman, J., Petty, C. R., Monuteaux, M. C., Mick, E., Parcell, T., Westerberg, D., \& Faraone, S. V. (2008). The longitudinal course of comorbid oppositional defiant disorder in girls with ADHD: Findings from a controlled 5-year prospective longitudinal follow-up study. Journal of Developmental \& Behavioral Pediatrics, 29, 501-507.

Choca, J. P. (2004). Interpretive guide to the Millon Clinical Multiaxial Inventory. Washington, DC: American Psychological Association.

Choca, J. P., \& Grossman, S. D. (2015). Evolution of the Millon Clinical Multiaxial Inventory. Journal of Personality Assessment, 97, 541-549.

Coid, J., Yang, M., Tyrer, P., Roberts, A., \& Ullrich, S. (2006). Prevalence and correlates of personality disorder in Great Britain. The British Journal of Psychiatry: The Journal of Mental Science, 188, 423-431.

Cook, J., Knight, E., Hume, I., \& Qureshi, A. (2014). The selfesteem of adults diagnosed with attention-deficit/hyperactivity disorder (ADHD): A systematic review of the literature. Attention Deficit and Hyperactivity Disorders, 6, 249-268.

Craig, R. J. (2005). New directions in interpreting the Millon Clinical Multiaxial Inventory-III (MCMI-III). New York, NY: John Wiley.

Cuevas, L., García, L. F., Aluja, A., \& García, Ó. (2008). Exploratory and confirmatory factorial structure of the MCMI-III Personality Disorders: Overlapping versus nonoverlapping scales. The European Journal of Psychiatry, 22(2), 59-68.

Dowson, J. H. (2008). Characteristics of adults with attentiondeficit/hyperactivity disorder and past conduct disorder. Acta Psychiatrica Scandinavica, 117, 299-305.

Edvinsson, D., Lindström, E., Bingefors, K., Lewander, T., \& Ekselius, L. (2013). Gender differences of axis I and II comorbidity in subjects diagnosed with attention-deficit hyperactivity disorder as adults. Acta Neuropsychiatrica, 25, 165-174.

Faraone, S. V., Kunwar, A., Adamson, J., \& Biederman, J. (2009) Personality traits among ADHD adults: Implications of lateonset and subthreshold diagnoses. Psychological Medicine, 39, 685-693.

Faraone, S. V., Sergeant, J., Gillberg, C., \& Biederman, J. (2003). The worldwide prevalence of ADHD: Is it an American condition? World Psychiatry, 2, 104-113.

Fayyad, J., Sampson, N. A., Hwang, I., Adamowski, T., AguilarGaxiola, S., Al-Hamzawi, A., . . . WHO World Mental Health Survey Collaborators. (2017). The descriptive epidemiology of DSM-IV Adult ADHD in the World Health Organization World Mental Health Surveys. Attention Deficit and Hyperactivity Disorders, 9, 47-65.

Fossati, A., Novella, L., Donati, D., Donini, M., \& Maffei, C. (2002). History of childhood attention deficit/hyperactivity disorder symptoms and borderline personality disorder: A controlled study. Comprehensive Psychiatry, 43, 369-377.

Grant, B. F., Stinson, F. S., Dawson, D. A., Chou, S. P., Ruan, W. J., \& Pickering, R. P. (2004). Co-occurrence of 12-month alcohol and drug use disorders and personality disorders in the United States: Results from the National Epidemiologic Survey on Alcohol and Related Conditions. Archives of General Psychiatry, 61, 361-368. 
Gudjonsson, G. H., Wells, J., \& Young, S. (2012). Personality disorders and clinical syndromes in ADHD prisoners. Journal of Attention Disorders, 16, 304-313.

Hamzeloo, M., Mashhadi, A., \& Salehi Fadardi, J. (2016). The prevalence of ADHD and comorbid disorders in Iranian adult male prison inmates. Journal of Attention Disorders, 20, 590 598.

Harpin, V., Mazzone, L., Raynaud, J. P., Kahle, J., \& Hodgkins, P. (2016). Long-term outcomes of ADHD: A systematic review of self-esteem and social function. Journal of Attention Disorders, 20, 295-305. doi:10.1177/1087054713486516

Harpold, T., Biederman, J., Gignac, M., Hammerness, P., Surman, C., Potter, A., \& Mick, E. (2007). Is oppositional defiant disorder a meaningful diagnosis in adults? Results from a large sample of adults with ADHD. The Journal of Nervous and Mental Disease, 195, 601-605. doi:10.1097/ NMD.0b013e318093f448

Hirsch, O., Chavanon, M., Riechmann, E., \& Christiansen, H. (2018). Emotional dysregulation is a primary symptom in adult attention-deficit/hyperactivity disorder (ADHD). Journal of Affective Disorders, 232, 41-47. doi:10.1016/j. jad.2018.02.007

Kajimura, S., Kochiyama, T., Nakai, R., Abe, N., \& Nomura, M. (2016). Causal relationship between effective connectivity within the default mode network and mind-wandering regulation and facilitation. NeuroImage, 133(suppl. C), 21-30.

Kane, M. J., Meier, M. E., Smeekens, B. A., Gross, G. M., Chun, C. A., Silvia, P. J., \& Kwapil, T. R. (2016). Individual differences in the executive control of attention, memory, and thought, and their associations with schizotypy. Journal of Experimental Psychology. General, 145, 1017-1048. doi:10. 1037/xge0000184

Kessler, R. C., Adler, L., Ames, M., Demler, O., Faraone, S., Hiripi, E., . . W Walters, E. E. (2005). The World Health Organization Adult ADHD Self-Report Scale (ASRS): A short screening scale for use in the general population. Psychological Medicine, 35, 245-256.

Klassen, L. J., Katzman, M. A., \& Chokka, P. (2010). Adult ADHD and its comorbidities, with a focus on bipolar disorder. Journal of Affective Disorders, 124, 1-8.

Kooij, J. J. S. (2006). ADHD in adults: Clinical studies on assessment and treatment. Amsterdam, The Netherlands: Harcourt Book Publishers. Retrieved from http://repository.ubn.ru.nl/ handle/2066/49720

Kooij, J. J. S. (2012). Adult ADHD: Diagnostic assessment and treatment. Springer Science \& Business Media. London: Springer London.

Kooij, J. J. S., Huss, M., Asherson, P., Akehurst, R., Beusterien, K., French, A., . . . Hodgkins, P. (2012). Distinguishing comorbidity and successful management of adult ADHD. Journal of Attention Disorders, 16(Suppl. 5), 3S-19S.

Matthies, S., \& Philipsen, A. (2016). Comorbidity of personality disorders and adult attention deficit hyperactivity disorder (ADHD)-Review of recent findings. Current Psychiatry Reports, 18, Article 33.

May, B., \& Bos, J. (2000). Personality characteristics of ADHD adults assessed with the Millon Clinical Multiaxial Inventory-II: Evidence of four distinct subtypes. Journal of Personality Assessment, 75, 237-248.
McKinney, A. A., Canu, W. H., \& Schneider, H. G. (2013). Distinct ADHD symptom clusters differentially associated with personality traits. Journal of Attention Disorders, 17, 358-366.

Miller, C. J., Flory, J. D., Miller, S. R., Harty, S. C., Newcorn, J. H., \& Halperin, J. M. (2008). Childhood attention-deficit/ hyperactivity disorder and the emergence of personality disorders in adolescence: A prospective follow-up study. The Journal of Clinical Psychiatry, 69, 1477-1484.

Millon, T., \& Grossman, S. D. (2007a). Moderating severe personality disorders: A personalized psychotherapy approach. Hoboken, NJ: John Wiley.

Millon, T., \& Grossman, S. D. (2007b). Overcoming resistant personality disorders: A personalized psychotherapy approach. Hoboken, NJ: John Wiley.

Newark, P. E., Elsässer, M., \& Stieglitz, R.-D. (2016). Selfesteem, self-efficacy, and resources in adults with ADHD. Journal of Attention Disorders, 20, 279-290.

Philipsen, A., Limberger, M. F., Lieb, K., Feige, B., Kleindienst, N., Ebner-Priemer, U., . . . Bohus, M. (2008). Attentiondeficit hyperactivity disorder as a potentially aggravating factor in borderline personality disorder. The British Journal of Psychiatry, 192, 118-123.

Ramos-Quiroga, J. A., Nasillo, V., Richarte, V., Corrales, M., Palma, F., Ibáñez, P., . . Kooij, J. J. (2019). Criteria and concurrent validity of DIVA 2.0: A semi-structured diagnostic interview for adult ADHD. Journal of Attention Disorders, 23(10), 1126-1135. doi:10.1177/1087054716646451

Salavera, C., Antoñanzas, J. L., Bustamante, J. C., Carrón, J., Usán, P., Teruel, P., . . . Cerra, R. (2014). Comorbidity of attention deficit hyperactivity disorder with personality disorders in homeless people. BMC Research Notes, 7 , Article 916.

Salgado, C. A. I., Bau, C. H. D., Grevet, E. H., Fischer, A. G., Victor, M. M., Kalil, K. L., . . Belmonte-de-Abreu, P. (2009). Inattention and hyperactivity dimensions of ADHD are associated with different personality profiles. Psychopathology, 42, 108-112.

Seli, P., Smallwood, J., Cheyne, J. A., \& Smilek, D. (2015). On the relation of mind wandering and ADHD symptomatology. Psychonomic Bulletin \& Review, 22, 629-636.

Strack, S., \& Millon, T. (2007). Contributions to the dimensional assessment of personality disorders using Millon's model and the Millon Clinical Multiaxial Inventory (MCMIIII). Journal of Personality Assessment, 89, 56-69. doi:10. 1080/00223890701357217

Torgersen, S., Kringlen, E., \& Cramer, V. (2001). The prevalence of personality disorders in a community sample. Archives of General Psychiatry, 58, 590-596.

Tyrer, P., Reed, G. M., \& Crawford, M. J. (2015). Classification, assessment, prevalence, and effect of personality disorder. The Lancet, 385, 717-726.

van Dijk, F., Lappenschaar, M., Kan, C., Verkes, R. J., \& Buitelaar, J. (2011). Lifespan attention deficit/hyperactivity disorder and borderline personality disorder symptoms in female patients: A latent class approach. Psychiatry Research, 190, 327-334.

Wise, E. A., Streiner, D. L., \& Walfish, S. (2010). A review and comparison of the reliabilities of the MMPI-2, MCMI-III, and PAI presented in their respective test manuals. Measurement 
and Evaluation in Counseling and Development, 42, 246-254. doi:10.1177/0748175609354594

Wright, A. G. C. (2017). The current state and future of factor analysis in personality disorder research. Personality Disorders, 8, 14-25. doi:10.1037/per0000216

Zennaro, A., Ferracuti, S., Lang, M., \& Sanavio, E. (2008). The Millon Clinical Multiaxial Inventory-III (MCMI-III), Adattamento Italiano. Firenze, Italy: Giunti OS.

\section{Author Biographies}

Francesco Oliva, MD, is an assistant professor of psychiatry at the Department of Clinical and Biological Sciences, University of Turin, Italy.
Chiara Mangiapane, MD, is a postgraduate from University of Turin, Italy.

Gabriele Nibbio, MD, is a psychiatry resident at the Department of Clinical and Experimental Sciences, University of Brescia, Italy.

Alberto Portigliatti Pomeri, MD, is a psychiatry resident at the Department of Neurosciences "Rita Levi Montalcini”, University of Turin, Italy.

Giuseppe Maina, MD is a full professor of psychiatry at the Department of Neurosciences "Rita Levi Montalcini”, University of Turin and the director of the Psychiatric unit, San Luigi Gonzaga University Hospital, University of Turin, Italy. 